\title{
Approaches to screening for latent tuberculosis infection in patients with immune-mediated disease prior to commencement of biologics
}

\author{
LS Tam *, MD \\ Division of Rheumatology, Department of Medicine and Therapeutics, The Chinese University of Hong Kong, Hong Kong \\ *Corresponding author: Istam@cuhk.edu.hk
}

Hong Kong Med J 2020;26:93-4

https://doi.org/10.12809/hkmi205096

With the rapid advancement in immunology, physicians caring for patients with immunemediated inflammatory diseases may find they have too many treatment options. Nevertheless, there are still problems with increased risk of infection associated with the use of biological agents. Tuberculosis remains one of the most important infections in areas where it is endemic, such as Hong Kong, where it has a standardised incidence ratio of 10.91 (95\% confidence interval $[95 \% \mathrm{CI}]=8.00-13.82$ ) in patients with immune-mediated inflammatory diseases treated with biologics compared with the general population. ${ }^{1}$ The diagnosis and treatment of individuals with latent tuberculosis infection (LTBI) who are at higher risk of developing active tuberculosis is an important step prior to commencement of biologics. However, diagnosis of LTBI is problematic because the tuberculin skin test (TST) has several limitations. False-positive results may be caused by exposure to non-tuberculosis mycobacteria or prior Bacillus Calmette-Guérin (BCG) vaccination. Falsenegative results due to inter-observer variability and the booster effect could reduce the efficiency of a strategy of targeted use of the TST and treatment of LTBI. ${ }^{2,3}$ In patients with rheumatoid arthritis, impaired cell-mediated immunity may result in false-negative TST, regardless of the presence of immunosuppressive medications. ${ }^{4}$ Furthermore, corticosteroids or methotrexate may decrease TST sensitivity. ${ }^{5}$ Notably, patients with psoriasis may develop new psoriatic lesions at the site of minor skin trauma (the Koebner phenomenon), which may be confused with a positive TST. ${ }^{6}$

Interferon $\gamma$ release assay (IGRA) has provided an alternative method for diagnosing LTBI based on TST. As IGRA measures interferon $\gamma$ released by $\mathrm{T}$ cells after stimulation with specific tuberculosis antigens, it does not cross-react with BCG and is free from false-positive results in vaccinated individuals. ${ }^{7}$ It has been shown to have a superior sensitivity and specificity than TST in the general population. $^{8}$ A recent meta-analysis showed that patients receiving immunosuppressive therapy were less likely to have a positive IGRA result (odds ratio $[\mathrm{OR}]=0.66,95 \% \mathrm{CI}=0.53-0.83, \mathrm{I}^{2}=23 \%$ ) than were patients not receiving immunosuppressive therapy.
This is especially so in patients receiving anti-tumour necrosis factor (anti-TNF) treatment $(\mathrm{OR}=0.50$, 95\% CI=0.29-0.88). The use of immunosuppressive therapy was also associated with a lower rate of positive TST result $(\mathrm{OR}=0.51,95 \% \mathrm{CI}=0.42-0.61){ }^{9}$

All patients who are candidates for biologic therapy with anti-TNF- $\alpha$ agents should undergo LTBI screening, and ideally should be screened at the time of diagnosis of an immune or inflammatory condition before starting on any immunosuppressive medications. This avoids confounding of screening tests by concomitant steroids and acknowledges the tuberculosis risk intrinsic to some immunemediated diseases and the risk associated with nonbiologic disease-modifying antirheumatic drugs. Screening should consist of a careful history as well as TST, IGRA, and chest radiography. A systematic review of clinical practice guidelines recommended either or both the TST and the IGRA for screening. ${ }^{10}$ The recommended choice of screening modalities and their frequency were reliant on test availability and costs.

As illustrated by the study from Tang et al in this issue of the Hong Kong Medical Journal, ${ }^{11}$ there were significantly more patients with tuberculosis in the single test group (mostly TST) than in the dual test group (9 [7.4\%] vs 1 [1.04\%]; $\mathrm{P}=0.045)$. Another report has raised the concern that TST as the only screening test for LTBI prior to antiTNF therapy was likely inadequate. ${ }^{12}$ Whether IGRA testing or TST have different predictive ability in discriminating who will progress to active tuberculosis is controversial. ${ }^{13,14}$ Data from Tang et al and others have also highlighted the discrepancies among IGRA assays and between IGRA and TST, ${ }^{15-17}$ making reliance on any single test unadvisable given the magnitude of the tuberculosis risk in this population.

In Hong Kong, because TST is widely available and economical, sequential testing may be considered: first a TST and, if negative (or $<10 \mathrm{~mm}$ ), an IGRA. Either a TST $>10 \mathrm{~mm}$ or a positive IGRA should be considered a positive screen; an indeterminate IGRA should be repeated. However, more studies are needed before we can be confident that this is the optimal screening strategy. 


\section{Author contributions}

The author contributed to concept, analysis or interpretation of data, drafting of the manuscript, and critical revision of the manuscript for important intellectual content. The author contributed to the manuscript, approved the final version for publication, and takes responsibility for its accuracy and integrity.

\section{Conflicts of interest}

The author has disclosed no conflicts of interest.

\section{References}

1. Wang X, Wong SH, Wang XS, et al. Risk of tuberculosis in patients with immune-mediated diseases on biological therapies: a population-based study in a tuberculosis endemic region. Rheumatology (Oxford) 2019;58:803-10.

2. Sepulveda RL, Ferrer X, Latrach C, Sorensen RU. The influence of Calmette-Guérin bacillus immunization on the booster effect of tuberculin testing in healthy young adults. Am Rev Respir Dis 1990;142:24-8.

3. Wang L, Turner MO, Elwood RK, Schulzer M, FitzGerald JM. A meta-analysis of the effect of Bacille Calmette Guérin vaccination on tuberculin skin test measurements. Thorax 2002;57:804-9.

4. Ponce de Leon D, Acevedo-Vasquez E, Alvizuri S, et al. Comparison of an interferon-gamma assay with tuberculin skin testing for detection of tuberculosis (TB) infection in patients with rheumatoid arthritis in a TB-endemic population. J Rheumatol 2008;35:776-81.

5. Kim EY, Lim JE, Jung JY, et al. Performance of the tuberculin skin test and interferon-gamma release assay for detection of tuberculosis infection in immunocompromised patients in a BCG-vaccinated population. BMC Infect Dis 2009;9:207.

6. Sivamani RK, Goodarzi H, Garcia MS, et al. Biologic therapies in the treatment of psoriasis: a comprehensive evidence-based basic science and clinical review and a practical guide to tuberculosis monitoring. Clin Rev Allergy Immunol 2013;44:121-40.

7. Pai M, Zwerling A, Menzies D. Systematic review: Tcell-based assays for the diagnosis of latent tuberculosis infection: an update. Ann Intern Med 2008;149:177-84.

8. Chang KC, Leung CC. Systematic review of interferon- gamma release assays in tuberculosis: focus on likelihood ratios. Thorax 2010;65:271-6.

9. Wong $\mathrm{SH}, \mathrm{Gao} \mathrm{Q}, \mathrm{Tsoi} \mathrm{KK}$, etal.Effect ofimmunosuppressive therapy on interferon $\gamma$ release assay for latent tuberculosis screening in patients with autoimmune diseases: a systematic review and meta-analysis. Thorax 2016;71:6472.

10. Hasan T, Au E, Chen S, Tong A, Wong G. Screening and prevention for latent tuberculosis in immunosuppressed patients at risk for tuberculosis: a systematic review of clinical practice guidelines. BMJ Open 2018;8:e022445.

11. Tang I, So H, Luk L, et al. Comparison of single and dual latent tuberculosis screening strategies before biologic and targeted therapy in patients with rheumatic diseases: a retrospective cohort study. Hong Kong Med J 2020;26:1119.

12. Raval A, Akhavan-Toyserkani G, Brinker A, Avigan M. Brief communication: characteristics of spontaneous cases of tuberculosis associated with infliximab. Ann Intern Med 2007;147:699-702.

13. Diel R, Loddenkemper R, Nienhaus A. Predictive value of interferon $-\gamma$ release assays and tuberculin skin testing for progression from latent tb infection to disease state: a meta-analysis. Chest 2012;142:63-75.

14. Abubakar I, Drobniewski F, Southern J, et al. Prognostic value of interferon- $\gamma$ release assays and tuberculin skin test in predicting the development of active tuberculosis (UK PREDICT TB): a prospective cohort study. Lancet Infect Dis 2018;18:1077-87.

15. Mariette X, Baron G, Tubach F, et al. Influence of replacing tuberculin skin test with ex vivo interferon $\gamma$ release assays on decision to administer prophylactic antituberculosis antibiotics before anti-TNF therapy. Ann Rheum Dis 2012;71:1783-90.

16. Bocchino M, Matarese A, Bellofiore B, et al. Performance of two commercial blood IFN-gamma release assays for the detection of Mycobacterium tuberculosis infection in patient candidates for anti-TNF-alpha treatment. Eur J Clin Microbiol Infect Dis 2008;27:907-13.

17. Hsia EC, Schluger N, Cush JJ, et al. Interferon- $\gamma$ release assay versus tuberculin skin test prior to treatment with golimumab, a human anti-tumor necrosis factor antibody, in patients with rheumatoid arthritis, psoriatic arthritis, or ankylosing spondylitis. Arthritis Rheum 2012;64:2068-77. 